Relations industrielles

Industrial Relations

\title{
L'Analyse des conventions collectives de travail Aspects méthodologiques
}

\section{Félix Quinet}

Volume 17, numéro 1, janvier 1962

URI : https://id.erudit.org/iderudit/1021648ar

DOI : https://doi.org/10.7202/1021648ar

Aller au sommaire du numéro

Éditeur(s)

Département des relations industrielles de l’Université Laval

ISSN

0034-379X (imprimé)

1703-8138 (numérique)

Découvrir la revue

Citer cet article

Quinet, F. (1962). L'Analyse des conventions collectives de travail : aspects méthodologiques. Relations industrielles / Industrial Relations, 17(1), 3-10. https://doi.org/10.7202/1021648ar

\section{Résumé de l'article}

L'analyse des conventions collectives de travail effectuée soit par le Ministère fédéral du travail ou d'autres institutions privées est une tâche aussi difficile qu'importante. Elle soulève des problèmes de méthode qu'il est bon de connaître pour pouvoir évaluer les conclusions. M. Quinet explique ici les méthodes utilisées à la Division de l'économie et de recherches du Ministère fédéral du Travail. M. Jean-Réal Cardin commente le travail de M. Quinet.
Tous droits réservés (C Département des relations industrielles de l’Université Laval, 1962
Ce document est protégé par la loi sur le droit d'auteur. L’utilisation des services d'Érudit (y compris la reproduction) est assujettie à sa politique d'utilisation que vous pouvez consulter en ligne.

https://apropos.erudit.org/fr/usagers/politique-dutilisation/ 


\section{L'Analyse des conventions collectives de travail}

\section{Aspects méthodologiques}

\section{Félix Quinet}

L'analyse des conventions collectives de travail effectuée soit par le Ministère fédéral du travail ou d'autres institutions privées est une tâche aussi difficile qu'importante. Elle soulève des problèmes de méthode qu'il est bon de connaître pour pouvoir évaluer les conclusions. M. Quinet explique ici les méthodes utilisées à la Division de léconomie et de recherches du Ministère fédéral du Travail. $M$. Jean-Réal Cardin commente le travail de M. Quinet.

\section{A. Introduction}

L'importance du travail de recherche et d'analyse dans le domaine des conventions collectives peut être difficilement niée. ${ }^{1}$ Citons quelques chiffres. D’après l'enquête menée en 1960 par le Ministère fédéral du Travail sur les conditions de travail dans l'industrie canadienne, ${ }^{2}$ il ressort que 66 p. 100 des travailleurs d'usine dans l'industrie manufacturière sont couverts par des conventions collectives. Dans l'industrie minière (secteur métaux), la proportion est de 82 p. 100, tandis qu'elle atteint 89 p. 100 dans le secteur « charbon » de la même industrie. Pour le Canada tout entier, on peut dire que les conditions de travail d'environ 1,500,000 travailleurs sont déterminées par voie de négociation collective. Il va sans dire que ces conditions de travail exercent à leur tour une influence sur celles de Yensemble des travailleurs, qu'ils soient syndiqués ou non.

QUINET, FÉLLx, M.R.I. (Montréal). Attaché à la Division de l'économie et des recherches, Ministère fédéral du Travail, Ottawa.

(1) Les remarques présentées ici sont personnelles et ne reflètent pas nécessairement les vues du Ministère du Travail du Canada. L'auteur est attaché à la Division de l'économie et des recherches de ce Ministère.

(2) Voir Working Conditions in Canadian Industry 1960, Economics and Research Branch, Department of Labour, Canada, pp. 66, 92, 93. 


\section{Les conventions collectives et le milieu social}

Il est clair aussi que les conventions collectives, de par leur contenu, exercent une profonde influence sur la vie sociale et familiale des travailleurs. Par exemple, la semaine de quarante heures pose des problèmes d'utilisation des loisirs, ou des problèmes de double emploi. On remarque d'ailleurs que le mouvement syndical accorde au problème du double emploi une attention de plus en plus grande.

Certes, si les conventions collectives exercent une influence sur le milieu social, elles sont également influencées par lui. Il est permis ici de poser quelques questions: l'attitude plutôt hostile d'une collectivité à l'égard du travail du dimanche peut-elle se traduire, dans la convention collective, par une majoration du taux de salaire payé pour le travail du dimanche? Ou encore, les sentiments religieux d'une collectivité peuvent-ils se manifester, dans le contrat de travail, par un nombre plus élevé de jours de fête chômés et payés? Par ailleurs, la solidité de la famille en tant que cellule sociale peut-elle se traduire par une certaine libéralité dans les dispositions du contrat accordant, par exemple, des congés rémunérés à l'occasion de la maladie ou du décès de membres de la famille du travailleur? Ce sont là des facteurs difficiles à mesurer exactement, mais qui ne sont pas à exclure. Il est meme possible que des idées politiques puissent influencer le contenu du contrat collectif: une convention signée dans une grande ville industrielle stipule qu'un travailleur ne peut être congédié s'il s'abstient de travailler le premier mai. ${ }^{3}$

\section{B. Lo préporation d'un code d'analyse de conventions collectives}

Quoi qu'il en soit, il est évident que le travail de recherches en conventions collectives nous permet d'avoir non seulement une idée précise des conditions de travail dans l'industrie canadienne, mais aussi une idée concrète du mode de vie d'un vaste secteur de notre collectivité.

Le Service des conventions collectives du Ministère fédéral du Travail, possède la copie de quelque 6,000 contrats de travail signés au Canada. Ces contrats sont classés par industrie suivant la méthode de classification industrielle en vigueur au Bureau fédéral de la Statistique. Ils sont également classés par province. Le Service analyse ces contrats au moyen d'un code qui doit être complété et revisé fréquemment.

(3) Je laisse délibérément de côté les facteurs purement économiques qui, à eux seuls, nécessiteraient toute une étude. 


\section{Nécessité de définitions claires}

La préparation d'un code d'analyse de conventions collectives est un travail de longue haleine. Il suppose d'abord la lecture de nombreuses conventions collectives signées dans des industries les plus diverses possible. Cela peut paraitre élémentaire, mais c'est primordial. D’ailleurs, la première conclusion à laquelle cette lecture conduit, c'est qu'il ne faut pas se laisser dominer par la terminologie ou le style des conventions collectives. La même expression peut vouloir dire une chose dans un contrat, et une chose tout à fait différente dans un autre. Par exemple, l'expression « indemnité de fin d'emploi » (Severance Pay) peut signifier des choses bien différentes. Dans certains contrats, on appelle indemnité de fin d'emploi ce qui n'est en réalité qu'un versement tenant lieu d'avis de mise à pied. Dans d'autres contrats, l'expression vise les différentes sommes d'argent qui sont dues aux travailleurs mis à pied (par exemple, crédits de vacances et crédits de maladie non utilisés, etc....). Enfin, dans d'autres contrats, l'expression englobe exclusivement des paiements spéciaux qui sont versés aux travailleurs perdant leur emploi pour une raison indépendante de leur volonté, travailleurs dont on ne s'attend pas qu'ils soient réembauchés. En ce qui nous concerne, c'est cette dernière définition que nous utilisons, en ayant évidemment soin de ne pas perdre de vue les divers éléments que nous avons éliminés de la définition.

Les différents sens donnés à l'expression indemnité de fin d'emploi (et ce n'est là qu'un exemple parmi bien d'autres!) nous montrent bien que les termes et expressions en usage dans le domaine de la négociation collective doivent être définis le plus clairement possible. Tout code d'analyse des contrats collectifs doit d'ailleurs reposer sur des définitions claires. Une fois les termes définis, il est plus aisé de diviser la convention collective en secteurs d'analyse. Ces secteurs seront ceux du code. ${ }^{4}$ Et de nombreux secteurs du code engloberont un éventail de possibilités. ${ }^{5}$ Les différents articles du secteur s'excluent mutuellement. En

(4) La convention collective a été divisée en près de 80 secteurs d'analyse. A titre d'exemples, en voici quelques-uns: secteurs concernant respectivement l'indernnité de présence; l'indemnité de fin d'emploi; les dispositions spéciales relatives à la rémunération du travail accompli le dimanche ou le $7 \mathrm{e}$ jour remplaçant le dimanche (le travail est régulièrement prévu); les congés statutaires payés; les droits spéciaux d'ancienneté pour dirigeants syndicaux; etc.

(5) Par exemple, le secteur concernant la « Sécurité syndicale - Qualité de Membre du Syndicat pourrait englober les possibilités suivantes: 1) atelier fermé; 2) atelier syndical (affiliation obligatoire pour tous les travailleurs); 3) atelier syndical modifié (affiliation obligatoire pour les travailleurs nouveaux seulement, maintien d'affiliation pour les autres); 4) atelier syndical modifié (affiliation obligatoire pour les travailleurs nouveaux seulement, sans maintien d'affiliation pour les autres); 5) maintien d'affiliation. 
d'autres termes, une convention ne pourra être codifiée qu'une fois sous chaque secteur. Les contrats collectifs pourront ainsi être passés au crible, sous tous leurs aspects.

\section{L'utilité de définitions générales et abstraites}

Il faut toutefois remarquer que certaines dispositions de contrats collectifs nécessitent, afin d'être correctement analysées, un certain travail de nature conceptuelle. La définition de termes techniques ne suffit pas dans ce cas. Il faut une définition générale et même abstraite de laquelle on part pour faire le travail d'analyse. Cette remarque s'applique, par exemple, aux « majorations du salaire accordées pour du temps de travail \. $^{\circ}$

\section{Evolution du contenu des conventions collectives}

Une fois le code d'analyse établi, la pire chose qui puisse arriver, c'est qu'on considère le travail terminé. En fait, il ne fait que commencer. Il ne faut jamais perdre de vue que les conventions collectives ne sont pas des textes statiques, mais des documents qui épousent une réalité toujours changeante. Il est nécessaire que ceux qui analysent les conventions collectives restent constamment aux aguets: certaines dispositions ont tendance à disparaître tandis que d'autres se font de plus en plus fréquentes. De nouvelles dispositions, émanant de situations ou d'attitudes nouvelles, font également leur apparition. Il est alors nécessaire de modifier ou de compléter le code. Citons un exemple. Des articles de convention concernant le statut personnel du travailleur vis-à-vis l'organisation syndicale et vis-à-vis son emploi sont maintenant assez fréquemment remarqués en marge des clauses de sécurité syndicale stipulant que l'appartenance au syndicat est une condition d'emploi et/ou que les travailleurs qui sont membres d'un syndicat donné seront embauchés de préférence à ceux qui n'en sont pas membres. Nous avons donc créé un nouveau secteur d'analyse s'in-

(6) Par exemple, on pourrait définir la majoration du salaire pour du temps de travail comme étant la somme d'argent versée au travailleur en plus du salaire régulier (straight time pay) qu'il reçoit pour du temps de travail; on pourrait aussi préciser que le travailleur peut avoir droit à cette majoration du salaire en raison de la nature du travail qu'il accomplit et/ou en raison du temps durant lequel il accomplit le travail. On pourrait ainsi analyser le contrat en se basant sur les distinctions suivantes: 1) majorations du salaire déterminées par la nature du travail accompli; 2) majorations du salaire déterminées par le temps durant lequel le travail est accompli, ces dernières majorations comprenant: a) la majoration du salaire pour du temps supplémentaire; b) la majoration du salaire pour le travail par poste (shift work); c) la majoration du salaire déterminée par des conditions autres que le temps supplémentaire et le travail par poste. 
titulant « Membre de l'organisation syndicale - Acquisition ou perte de la qualité de...». A titre d'exemple, voici trois articles qui tombent sous le nouveau secteur:

«The members of the . . . . . agree to operate preferential union shops... The provisions for preference herein require that the doors of the union shall be kept open for the reception of non-union workers.

Initiation fees and dues must be maintained at a reasonable rate and any applicant must be admitted who is not an offender against the Uuion, and who is eligible for membership under its rules. If any rule is passed that imposes unreasonable hardship, or that operates to bar desirable persons the matter may be brought before the Board of Arbitration for such remedy as it may deem advisable... 》

«...The Union agrees that any employee engaged by the Company to work in the bargaining unit who is satisfactory to the Company will be allowed to make application for membership in the said local union on payment of the regular initiation fees and monthly dues, and will not be denied membreship without just cause... 》

*...Whenever an employee is suspended or expelled, the Uuion will give the Company in writing the reason for such action... »

«The Union shall be the sole judge of the good standing of its members. Any employee who fails to become a member of the Union within the time limit set forth herein below or who fails to pay the required initiation fees and dues as prescribed by the Union or who shall hereafter cease to be a member of the Union in good standing shall be immediately discharged upon written notice from the Union to the Employer setting forth the reasons for the discharge. Expulsion or suspension of members by the Local Union shall be under regulations consistent with the constitution of the International Union. »

On notera qu'il y a dans ces trois articles matière à analyse et matière à nuances. Nous avons également créé un nouveau secteur consacré aux travailleurs âgés en raison de l'intérêt grandissant qui s'est manifesté à l'égard de cette catégorie de travailleurs. Ce secteur nous a d'ailleurs permis de préparer un article consacré aux Clauses de Conventions collectives visant les problèmes des travailleurs ágés. Voir Gazette du Travail, Vol. LIX, No 19, pp. 1063-1067, octobre 1959). Si notre Code nous a permis de faire notre étude sur les dispositions con- 
cernant les travailleurs âgés, cette étude nous a permis, elle, de «raffiner notre code. C'est ainsi qu'il me semble que la plupart des clauses concernant les travailleurs âgés peuvent être, jusqu'à nouvel ordre, groupées dans l'une ou dans plusieurs des cinq catégories suivantes: a) le contrat collectif peut stipuler qu'il n'y aura aucune discrimination en ce qui concerne l'embauchage, et/ou le maintien en emploi, et/ou la fin de l'emploi des travailleurs âgés; b) le contrat peut stipuler que les travailleurs âgés feront l'objet de considération particulière; toutefois le contrat ne fournit pas de détail ou sujet de cette considération; c) le contrat contient une disposition spéciale concernant le transfert du travailleur âgé à un emploi qui lui convient mieux; d) le contrat contient une disposition spéciale concernant le salaire qui peut être payé à un travailleur âgé; e) enfin, le contrat contient une disposition spéciale concernant l'ancienneté du travailleur âgé... Tout ceci pour dire que l'une des façons d'améliorer notre code, c'est de l'utiliser!

\section{Utilité du code d'analyse des contrats collectifs}

Ceci m'amène à décrire les usages que nous faisons du code d'analyse des conventions collectives. Ce code nous sert à produire des études de deux sortes: d'abord, des études générales portant sur tous les articles ou la plupart des articles des conventions collectives. (Exemple: Conventions collectives dans lindustrie manufacturiere au Canada, 1956. Voir Gazette du Travail, Vol. LVII, No 4, pp. 472-482, Avril 1957). Ces études ont évidemment pour but premier d'indiquer la fréquence des articles de convention dans une industrie, ou dans un secteur industriel donné. D’une étude générale à l'autre, nous sommes donc en mesure de connaître les changements qui ont affecté le contenu des conventions collectives. Ensuite, des études analytiques portant sur un article de convention, ou un secteur de convention. (Exemple: Clauses de conventions collectives visant les problèmes des travailleurs âgés. Voir Gazette du Travail, Vol. LIX, No 10, pp. 1063-1067, Octobre 1959). Dans ce cas, le code nous permet de découvrir l'article étudié dans les contrats que nous examinons. Le travail analytique vient après.

\section{Problèmes inhérents aux études d'une nature statistique}

Il faut dire ici que la préparation des études générales soulève certains problèmes: il est parfois difficile, et même impossible, d'établir la fréquence statistique de certains articles, en tenant compte de leur contenu. Par exemple, classer les articles de convention concernant les 
congés-maladie payés en tenant uniquement compte du nombre de jours disponibles par an, peut laisser de côté certaines conditions préalables à l'obtention de tels congés, ou certaines conditions entourant le paiement de ces congés. Par exemple, on peut avoir deux contrats qui, tous deux, vont stipuler que le travailleur au service de la Compagnie depuis un an pourra bénéficier de 8 jours de congés-maladie payés par an; toutefois, l'un des deux contrats peut stipuler que pour recevoir le paiement, le travailleur doit être malade plus de deux jours; s'il n'est malade que durant un ou deux jours, il ne recevra rien. Ceci n'est qu'un exemple choisi parmi de nombreux autres. Mais il nous montre que dans certains cas, nous sommes forcés de nous borner à signaler la présence des articles de convention, sans plus. Certes, il nous sera toujours possible dans nos études portant sur un article précis d'être plus analytiques et de considérer l'article étudié sous tous ses aspects.

En préparant les études générales, il ne faut non plus pas perdre de vue que ce sont les résultats de la négociation collective que nous analysons plutôt que l'influence qu'exerce la nature de l'industrie sur le contenu du contrat collectif. Je m'explique. Dans certaines industries (l'industrie minière, par exemple) l'existence de nombreux Comités de Sécurité découle autant de la nature de l'industrie que de la négociation collective. Dans d'autres industries (par exemple, des industries comprenant des occupations spécialisées) les clauses établissant l'ancienneté sur la base du département découlent, aussi, autant de la nature de l'industrie que de la négociation collective proprement dite. C'est ainsi qu'en constatant un plus grand nombre de Comités de Sécurité dans certaines industries que dans d'autres, il ne faudrait pas nécessairement conclure que les syndicats et les employeurs y sont plus soucieux de la sécurité du travailleur que dans les industries comptant moins de Comités de Sécurité. Cet exemple appelle une remarque d'ordre général: la fréquence de certains articles de conventions collectives ne peut, nécessairement, être valablement comparée d'une industrie à l'autre.

\section{Conclusion}

Ces dernières remarques, comme celles qui précèdent, visent à indiquer certains problèmes que soulève l'analyse des contrats collectifs. Certes, cette étude est loin d'être exhaustive, mais elle peut donner une idée des méthodes que nous suivons dans notre travail. Avant de terminer, il faut mentionner que, très souvent, ceux qui utilisent nos études sont aussi ceux qui les produisent: les employeurs et les travailleurs. Les contrats qu'ils négocient constituent l'objet de nos travaux. Si les 
négociateurs consultent nos études, il est possible qu'ils en retirent quelques éléments qu'ils voudront introduire dans leur propre contrat. Mais leur propre contrat, analysé comme les autres, sera peut-être utilisé par d'autres négociateurs. De toute façon, il est indéniable que les contrats collectifs s'influencent réciproquement. C'est là la preuve que le domaine des conventions collectives est un domaine vraiment dynamique; c'est pourquoi l'analyse des contrats collectifs est rarement facile et rarement routinière.

\section{Commentaires}

\section{Jean-Réal Cordin}

La convention collective, quoi qu'on en ait dit ou qu'on en dise encore aujourd'hui, demeure le pivot des relations industrielles en Amérique du Nord, l'instrument par excellence à l'aide duquel, dans les secteurs syndiqués de l'économie, les conditions de travail et d'emploi sont définies, le procédé selon lequel la vie entière de l'entreprise s'organise et se poursuit au jour le jour.

M. Quinet a voulu nous faire voir quelques aspects méthodologiques d'analyse des conventions collectives telles qu'elles s'expriment au Canada présentement. Ce faisant, il rejoint, avec le Ministère canadien du Travail, la préoccupation nécessaire que l'on retrouve chez d'autres organismes spécialisés dans les questions du travail, comme le Bureau international du Travail ou encore le Département du Travail des Etats-Unis qui a publié des études portant sur lanalyse des principales clauses de conventions collectives en vigueur chez nos voisins du Sud, ou en d'autres pays industrialisés.

L'analyse et l'appréciation des conventions collectives de travail prend d'autant plus d'importance dans le contexte nord-américain, que presque tous les aspects du statut des parties en présence, des droits respectifs de chacune d'elles, de leurs devoirs réciproques, des conditions de travail et d'emploi des travailleurs-individus, du règlement des conflits susceptibles de survenir dans l'administration des relations industrielles, ainsi qu'une large part de la sécurité sociale et du bien-être des salariés de l'industrie sont, en Amérique du Nord, donc au Canada, réglés au moyen de la convention de travail, de caractère privé et contractuel. 